\title{
Diversity and Abundance of Birds at Dhapchapak Riverine Forest, Dera Ismail Khan, Pakistan
}

\author{
Najam-un-Nisa ${ }^{1}$, Ruqia Bibi ${ }^{1}$, Balqees Riaz ${ }^{1}$, Bushra Khali1 ${ }^{1}$, Iqra Maheen ${ }^{1}$, Saima ${ }^{1}$, Uzma Islam Khan ${ }^{1}$ \\ and Inam Ullah ${ }^{1, *}$
}

${ }^{1}$ Department of Zoology, Govt girls degree college no 02, Dera Ismail Khan, Pakistan; ${ }^{2}$ College of Wildlife and Protected Area, Northeast Forestry University, Harbin 150040, P.R. China.

Abstract | This study aims to assess the avifauna diversity in Dhapchapak lake and forest areas. There is no
previous record of any detailed research on bird species in Dhapchapak lake and the forest area. The study
was conducted to estimate the diversity and abundance of avifauna species found in dhapchapak wetland and
forest during winter (2017-18 and 2018-19). As birds are the best indicator for environmental changes hence
the migratory birds fauna was observed during September to March of the year 2017-1019. Point transect
method was used to explore the avifauna diversity at study site. In current study, total of 13,933 birds belonging
to 39 species, 21 families were observed. Among these 39 species, 33 bird's species were terrestrial and the rest
of 21 bird's species were aquatic. In water birds species, Great Egret is the most abundant specie of 2017-18
year, while in 2018-19 year, most abundant specie is Little Cormorant. In forest birds species, most abundant
species of the study area were recorded as Little Cormorant and Northern Pintail. As migratory birds indicate
ecosystem condition, healthy birds species indicate healthy habitat, while declining birds indicate degraded
habitat. The objective of this study was to determine the bird species composition, species diversity, species
richness, species evenness and status of bird species.
Received | April 19, 2021; Accepted | July 07,2021; Published | November 06, 2021
*Correspondence | Inam Ullah, College of Wildlife and Protected Area, Northeast Forestry University, Harbin 150040, P. R. China; Email:
Inamullah3554@gmail.com
Citation | Nisa, N., R. Bibi, B. Riaz, B. Khalil, I. Maheen, Saima, U.I. Khan and I. Ullah. 2021. Diversity and abundance of birds at dhapchapak
riverine forest, Dera Ismail Khan, Pakistan. Journal of Innovative Sciences, 7(2): 236-243.
DOI | https://dx.doi.org/10.17582/journal.jis/2021/7.2.236.243
Keywords | Dhapchapak wetland, Waterfowls bird, Relative abundance, Diversity, Pakistan

\section{Introduction}

$\mathrm{T}$ he biosphere is a collection of a variety of ecological zones and each zone is according to the adaptation of species in it. Biological diversity has been greatly enhanced by a smooth interaction between ecosystems and species (Richard, 1998). Biodiversity can significantly be impacted by the quality of the ecological environment (Pei, 2018). The best-studied group of vertebrates on the planet is birds (Whelan et al., 2008). Birds have widespread occurrences; they are mobile and have many ecological roles (Daniel, 2011). Birds provide supporting services such as pollination, seed dispersal, water purification, and nutrient recycling (Brenner, 2010).

Wetland habitat structure has an impact on bird species composition (Wondiumwolde, 2018). Wetlands are defined as lands transitional between terrestrial and aquatic ecosystems. Wetlands serve as a home for a vast diversity of wildlife such as birds, mammals, fish, frogs, insects, plants (Buckton, 2007). Pakistan has the most extensive canal system, consists of 225 wetlands. Birds are noted as 9993 species worldwide, 2700 
species from Asia. However, more than 668 species from Pakistan. In Pakistan, $30 \%$ of avian species are long-distance migrants, $43 \%$ of species are oriented, or Palearctic $27 \%$ are winter visitors (Roberts, 1991). Cranes, ducks, falcons, flamingos, geese, swans, and waders are vital migratory avian species in Pakistan (Ali, 2005)

The underlying biodiversity is closely related to the forest ecosystem and its multifunctionality (Gamefeldt, 2013). Many organisms have been affected by the available forest area and water bodies. Birds diversity yet unknown in the forest and wetland of Dera Ismail Khan Dhapchapak, which is present at KPK and Punjab's borderline. This study aims to assess the avifauna diversity in Dhapchapak lake and forest areas. There is no previous record of any detailed research on bird species in Dhapchapak lake and the forest area. This study's objective was to determine the bird species composition, species diversity, species richness, species evenness, and status of bird species.

\section{Materials and Methods}

\subsection{Study site}

The study was conducted at Dhapchapak forest and wetland, situated at $\left(31.784703^{\circ} \mathrm{N}, 70.971812^{\circ} \mathrm{E}\right)$, the border of Khyber Pakhtunkhwa, and Punjab provinces of Pakistan, and is located at river Indus. The area is fertile and is rich in vegetation consisting of trees, herbs, and shrubs. This region serves as essential habitat for migratory birds and waterfowls because it consists of both forest and wetland. This study aims to learn about the avian biodiversity of this region. Dera Ismail Khan has a hot desert climate with sweltering summers and warm winters. Rain mainly falls in two distinct periods: in the late winter and early spring from February to April, and in the monsoon in July and August.

\subsection{Data collection}

Both the forest and wetland of Dhapchapak were surveyed to estimate the number of species of birds twice a day. The survey was carried out each month from September 2017 to March 2018 and September 2018 to March 2019. The study site was visited twice each month. For data collection, we used three methods, i.e., the Point count method, the Line transect method, and direct observation. The survey was conducted at dawn (6 am-8 am) and dusk (5 pm-7 $\mathrm{pm})$. During this time, birds actively perform their functions. For observation of bird species, binocular (42x) was used. The bird's photography was done with Nikon D7200 (sigma 150-600 mm lens). The location of the avian was recorded by BENQ GPS. Avian species were identified using keys of Woodcock (1980) and Kazmierczak (2000).

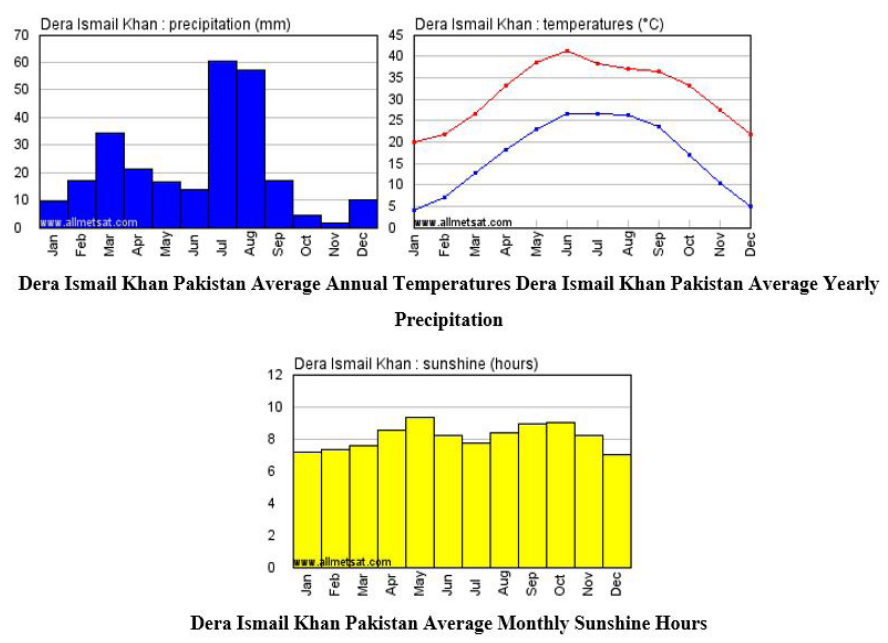

For the observation and calculation of birds, a pair of binoculars were used while staying apart distant. Indirect method observation, everything that can be seen or heard to be recorded. They were observing the number of individuals on the forest using the point count method while observing birds along/of wetland using the line-transect method.

\subsection{Data analysis}

The collected data were interned and stored in the Microsoft Excel Spreadsheet. We estimated the following parameters using that data:

(I) We determined the relative abundance of bird species of the area using the following equation

$$
(R . A)=n / N \quad \text {....(1) }
$$

Where;

R.A: Relative Abundance $\mathrm{n}=$ total number of individuals sighted of a bird species recorded during the surveys. $\mathrm{N}=$ total number of individuals sighted of all bird species recorded during the surveys.

(II) whenever we study avian fauna in any area, determining the diversity of bird species is always very significant. For this purpose, Shannon-Weiner Index $\left(\mathrm{H}^{\prime}\right)$ was calculated to know the species diversity based on species abundance using the following equation: 
$H^{\prime}=-\left[\Sigma P i^{*} L N(P i)\right]$

Where;

$\mathrm{H}^{\prime}=$ Diversity Index; $\mathrm{Pi}=$ proportion of each bird species in the sample; $\mathrm{LN}(\mathrm{Pi})=$ natural logarithm of this proportion.

(III) Evenness is another important feature of bird studies, determination of which is needed. Evenness compares the similarity of the different bird species population size in a whole sample (all bird species). We calculated the Evenness Index $\left(\mathrm{J}^{\prime}\right)$ using the following formula.

$$
J=H^{\prime} / H \max
$$

$\mathrm{H}^{\prime}=$ diversity index; $\mathrm{Hmax}=$ natural $\log$ of the total number of all bird species.

(IV) We calculated the Simpson Index (D), which is the probability of any two individuals drawn from noticeably large communities belonging to different species.

$$
D=1-\Sigma n(n-1) / N(N-1)
$$

$\mathrm{N}=$ total number of individuals of a bird species sighted during the surveys; $\mathrm{N}=$ total number of individuals of all bird species sighted during the surveys.

Point transects are like line transects. In the point transect method, several points are chosen within the study area, and each point is visited. The object detected from each point and then recorded, with their distances from the point. Ornithologists are the primary users of a method, which they called such sampling method as variable circular plot sampling.

Direct observation includes those techniques used in the wild that enables us to observe or hear everything that can be put in records in the true sense. Due to this primary reason, the method of automatic video recording is not helpful here compared to the direct observation method. Although this method is very efficient for analyzing some kinds of behaviors, it doesn't need that the observer follows the animal through its habitat.

\section{Result and Discussion}

hapchapak wetland and forest have a complex aquatic and terrestrial habitat, and due to this reason, they have a great variety of bird species. During the study, a total of 13,933 birds belonging to 39 species, 21 families were observed. Among these 39 species, 33 bird's species were terrestrial, and the rest of 21 bird's species were aquatic.

In waterfowls birds species, Family Anatidae was recorded to be the most diversified during the survey and represented by 11 species; after that, Family Ardeidae was recorded to be the $2^{\text {nd }}$ most diversified and denoted seven species. Family Scolopacidae represented by five species, while Families named Charadriidae and Podicipedidae were represented by three species (each). Families named Jacanidae,Laridae, Rallidae, Phoenicopteridae, Phalacrocoridae represented by 2 species (each). In water birds, five species are migratory birds, i.e., Anas crecca (622), Aythya ferina (661), Mareca penelope (639), Anas platyrbynchos (504), and Anas acuta (702). In waterbird species, Great Egret is the most abundant species of the 2017-18 year, having 183 in number, while in the 2018-19 year, the most abundant species is Little Cormorant having 200 in number. In the 2017-18 year, the most dominated and sub-dominant species are Great Egret (183) and Gadwall (174), respectively, while in the 2018-19 year, the most overpowered and sub-dominated species are Little Cormorant (200) and Gadwall (187), respectively.

In forest bird species, Family Muscicapidae was the most diversified during the survey and represented by four species. The $2^{\text {nd }}$ most diversified Families are Columbidae, Laniidae, Accipitridae, Apopidae, Cuculidae, Ploceidae, Meropidae, which were characterized by two species (each). Familes named Dicruridae, Upupidae,Falconidae, Sturnidae, Oriolidae, Corvidae, Coraciidae, Sylviidae, Nectariniidae, Pycnonotidae, Charadriidae, Passeridae were represented by 1 species (each). Forest birds were all considered residential birds. During this study, all birds' conservational status is the most minor concern, i.e., having a stable population. The most dominant species of forest birds is House Sparrow (369). The most abundant species of the study area were recorded as Little Cormorant (846) and Northern Pintail (702). $2^{\text {nd }}$ most abundant species of the study area are Red Wattled Lapwing (681), Cattle Egret (661), Common Pochard (661), Eurasian Wigeon (639), Intermediate Egret (638), Common Teal (622), Indian Pond Heron (508), Mallard (504). 
Table 1: Abundant bird species of dhapchapak wetland and forest.

\begin{tabular}{|c|c|c|c|c|}
\hline Species name & Total & $\mathbf{n} / \mathbf{N}$ & $\mathbf{H}^{\prime}$ & D \\
\hline Alpine Swift & 237 & 0.017009976 & -0.0693 & 0.000288 \\
\hline Asian Koel & 67 & 0.004808727 & -0.02567 & $2.28 \mathrm{E}-05$ \\
\hline Baya Weaver & 223 & 0.016005168 & -0.06618 & 0.000255 \\
\hline Bay-Backed Shrike & 93 & 0.006674801 & -0.03344 & $4.41 \mathrm{E}-05$ \\
\hline Black Drongo & 78 & 0.00559822 & -0.02903 & $3.09 \mathrm{E}-05$ \\
\hline Black headed gull & 317 & 0.02275174 & -0.08607 & 0.000516 \\
\hline Black-Breasted Weaver & 144 & 0.010335175 & -0.04725 & 0.000106 \\
\hline Black-necked grebe & 393 & 0.028206416 & -0.10065 & 0.000794 \\
\hline Blue-Cheeked Bee-Eater & 311 & 0.022321108 & -0.08487 & 0.000497 \\
\hline Cattle egret & 661 & 0.047441326 & -0.14461 & 0.002247 \\
\hline Common Buzzard & 53 & 0.003803919 & -0.02119 & $1.42 \mathrm{E}-05$ \\
\hline Common Hoopoe & 70 & 0.005024044 & -0.02659 & $2.49 \mathrm{E}-05$ \\
\hline Common Kestrel & 27 & 0.001937845 & -0.0121 & $3.62 \mathrm{E}-06$ \\
\hline Common Myna & 173 & 0.012416565 & -0.05449 & 0.000153 \\
\hline Common pochard & 661 & 0.047441326 & -0.14461 & 0.002247 \\
\hline Common sand piper & 139 & 0.009976315 & -0.04597 & $9.88 \mathrm{E}-05$ \\
\hline Common Stone Chat & 50 & 0.003588603 & -0.0202 & $1.26 \mathrm{E}-05$ \\
\hline Common teal & 622 & 0.044642216 & -0.1388 & 0.00199 \\
\hline Eurasian Golden Oriole & 66 & 0.004736955 & -0.02535 & $2.21 \mathrm{E}-05$ \\
\hline Eurasian wigeon & 639 & 0.045862341 & -0.14135 & 0.0021 \\
\hline Fork tailed Swift & 121 & 0.008684418 & -0.04122 & $7.48 \mathrm{E}-05$ \\
\hline Great black headed gull & 168 & 0.012057705 & -0.05327 & 0.000145 \\
\hline Greater Coucal & 57 & 0.004091007 & -0.0225 & $1.64 \mathrm{E}-05$ \\
\hline Green Bee-Eater & 158 & 0.011339984 & -0.0508 & 0.000128 \\
\hline Green shank & 134 & 0.009617455 & -0.04467 & $9.18 \mathrm{E}-05$ \\
\hline Grey Bush Chat & 126 & 0.009043279 & -0.04256 & $8.11 \mathrm{E}-05$ \\
\hline Grey heron & 263 & 0.01887605 & -0.07494 & 0.000355 \\
\hline House Crow & 187 & 0.013421374 & -0.05786 & 0.000179 \\
\hline House Sparrow & 369 & 0.026483887 & -0.09617 & 0.0007 \\
\hline Indian pond heron & 508 & 0.036460202 & -0.12074 & 0.001327 \\
\hline Indian Robin & 149 & 0.010694036 & -0.04853 & 0.000114 \\
\hline Indian Roller & 81 & 0.005813536 & -0.02993 & $3.34 \mathrm{E}-05$ \\
\hline Intermediate egret & 638 & 0.045790569 & -0.1412 & 0.002094 \\
\hline Little cormorant & 846 & 0.060719156 & -0.1701 & 0.003683 \\
\hline Little egret & 259 & 0.018588961 & -0.07408 & 0.000344 \\
\hline Little grebe & 612 & 0.043924496 & -0.13728 & 0.001926 \\
\hline Little ringed plover & 261 & 0.018732506 & -0.07451 & 0.00035 \\
\hline Little stint & 382 & 0.027416924 & -0.09861 & 0.00075 \\
\hline Mallard & 504 & 0.036173114 & -0.12007 & 0.001306 \\
\hline Northern pintail & 702 & 0.05038398 & -0.15055 & 0.002535 \\
\hline Orphean Warbler & 52 & 0.003732147 & -0.02087 & $1.37 \mathrm{E}-05$ \\
\hline Pheasant tail Jacana & 149 & 0.010694036 & -0.04853 & 0.000114 \\
\hline Purple heron & 205 & 0.014713271 & -0.06208 & 0.000215 \\
\hline Species name & Total & $\mathbf{n} / \mathbf{N}$ & $\mathbf{H}^{\prime}$ & D \\
\hline Purple Sunbird & 73 & 0.00523936 & -0.02751 & $2.71 \mathrm{E}-05$ \\
\hline Red Backed Shrike & 54 & 0.003875691 & -0.02152 & $1.47 \mathrm{E}-05$ \\
\hline Red Collared Dove & 69 & 0.004952272 & -0.02629 & $2.42 \mathrm{E}-05$ \\
\hline
\end{tabular}

\begin{tabular}{lllll} 
Red shank & 291 & 0.020885667 & -0.0808 & 0.000435 \\
Red wattled lapwing & 681 & 0.048876767 & -0.14753 & 0.002386 \\
Red-Vented bulbul & 132 & 0.009473911 & -0.04414 & $8.91 \mathrm{E}-05$ \\
Red-Wattled Lapwing & 115 & 0.008253786 & -0.03959 & $6.75 \mathrm{E}-05$ \\
$\begin{array}{l}\text { Rufous-Tailed Scrub } \\
\text { Robin }\end{array}$ & 98 & 0.007033661 & -0.03487 & $4.9 \mathrm{E}-05$ \\
Shikra & 37 & 0.002655566 & -0.01575 & $6.86 \mathrm{E}-06$ \\
Spanish Sparrow & 137 & 0.009832771 & -0.04545 & $9.6 \mathrm{E}-05$ \\
Spotted Dove & 90 & 0.006459485 & -0.03257 & $4.13 \mathrm{E}-05$ \\
White-tailed plover & 201 & 0.014426182 & -0.06115 & 0.000207 \\
& 13933 & 1 & 3.675948 & 0.968515 \\
\hline
\end{tabular}

The least abundant species of the survey were Asian Koel (67), Bay-Backed Shrike (93), Black Drongo (78), Common Buzzard (53), Common Hoopoe (70), Common Kestrel (27), Common Stone Chat (50), Eurasian Golden Oriole (66), Greater Coucal (57), Indian Roller (81), Orphean Warbler (52), Purple Sunbird (73), Red-Backed Shrike (54), Red Collared Dove (69), Rufous-Tailed Scrub Robin (98), Shikra (37).

In the present survey, we developed a checklist of bird diversity in dhapchapak wetland and forest and estimated their relative abundance. Most bird species recorded in dhapchapak forest and wetland belong to Family Phasianidae as it provides a suitable habitat for birds diversity to live and breed. Birds visit this site for drinking water and roosting on trees found around the study area. The total bird diversity of dhapchapak wetland and forest is 13933. In which waterfowls bird species recorded 10237 and forest birds are 3697.

Diversity is the critical component of an ecosystem, and among various organisms, birds have significant possession in an ecosystem because of noticeability and well appreciation by humans. Birds play an essential role in charming public attention to natural habitats. The variety in bird fauna in pristine habitats serves as an indicator of a habitat's natural status (Mahmood et al., 2021).

In a survey conducted during 2008-2009 at a place of kallarkahar lake, a total of 86 bird species which belong to 36 families, were recorded (Rais et al., 2011). Among 86 species, 29 water birds (in which 6 were ducks species and 23 were other waterbird species) were recorded at kalarkahar lake. During the January and February months of 2003, various 30 species of birds were recorded at such areas by Ali and Akhtar. At Taunsabarrage, a survey was conducted during 
Table 2: Birds diversity at dhapchapak wetland and forest.

\begin{tabular}{|c|c|c|c|c|c|c|}
\hline Scientific name & Species name & Family & $\begin{array}{l}\text { Conservation } \\
\text { status }\end{array}$ & $\begin{array}{l}\text { Distribu- } \\
\text { tion }\end{array}$ & Habitat & Abundance \\
\hline Tachymartis melba & Alpine Swift & Apodidae & Least concern & Residential & Terrestrial & sub-dominant \\
\hline Eudynamysscolopaceus & Asian Koel & Cuculidae & Least concern & Residential & Terrestrial & least abundant \\
\hline Ploceusphilippinus & Baya Weaver & Ploceidae & Least concern & Residential & Terrestrial & sub-dominant \\
\hline Laniusvittatus & Bay-Backed Shrike & Laniidae & Least concern & Residential & Terrestrial & least abundant \\
\hline Dicrurusmacrocercus & Black Drongo & Dicruridae & Least concern & Residential & Terrestrial & least abundant \\
\hline Chroicocephalusridibundus & Black headed gull & Laridae & Least concern & Residential & Aquatic & sub-dominant \\
\hline Ploceusbenghalensis & Black-Breasted Weaver & Ploceidae & Least concern & Residential & Terrestrial & least abundant \\
\hline Podicepsnigricollis & Black-necked grebe & Podicipedidae & Least concern & Residential & Aquatic & sub-dominant \\
\hline Meropssuperciliosus & Blue-Cheeked Bee-Eater & Meropidae & Least concern & Residential & Terrestrial & sub-dominant \\
\hline Bubulcus ibis & Cattle egret & Ardeidae & Least concern & Residential & Terrestrial & $2^{\text {nd }}$ most dominant \\
\hline Buteobuteo & Common Buzzard & Accipitridae & Least concern & Residential & Terrestrial & least abundant \\
\hline Upupaepops & Common Hoopoe & Upupidae & Least concern & Residential & Terrestrial & least abundant \\
\hline Falco tinnunculus & Common Kestrel & Falconidae & Least concern & Residential & Terrestrial & least abundant \\
\hline Acridotherestristis & Common Myna & Sturnidae & Least concern & Residential & Terrestrial & least abundant \\
\hline Aythyaferina & Common pochard & Anatidae & Least concern & Migratory & Aquatic & $2^{\text {nd }}$ most dominant \\
\hline Actitishypoleucos & Common sand piper & Scolopacidae & Least concern & Residential & Aquatic & least abundant \\
\hline Saxicolarubicola & Common Stone Chat & Muscicapidae & Least concern & Residential & Aquatic & least abundant \\
\hline Anascrecca & Common teal & Anatidae & Least concern & Migratory & Aquatic & $2^{\text {nd }}$ most dominant \\
\hline Oriolusoriolus & Eurasian Golden Oriole & Oriolidae & Least concern & Residential & Terrestrial & least abundant \\
\hline Marecapenelope & Eurasian wigeon & Anatidae & Least concern & Migratory & Aquatic & $2^{\text {nd }}$ most dominant \\
\hline Apuspacificus & Fork tailed Swift & Apodidae & Least concern & Residential & Terrestrial & least abundant \\
\hline Ichthyaetusichthyaetus & Great black headed gull & Laridae & Least concern & Residential & Aqua & least abundant \\
\hline Centropussinensis & Greater Coucal & Cuculidae & Least concern & Residential & strial & least abundant \\
\hline Meropsorientalis & Green Bee-Eater & Meropidae & Least concern & Residential & Terrestrial & least abundant \\
\hline Tringanebularia & Green shank & Scolopacidae & Least concern & Residential & Aquatic & least abundant \\
\hline Saxicolaferreus & Grey Bush Chat & Muscicapidae & Least concern & Residential & Terrestrial & least abundant \\
\hline Ardeacinerea & Grey heron & Ardeidae & Least concern & Residential & & \\
\hline Corvussplendens & House Crow & Corvidae & Least concern & Residential & Terrestrial & least abundant \\
\hline Passer domesticus & House Sparrow & Phasianidae & Least concern & Residential & Terrestrial & sub-dominant \\
\hline Ardeolagrayii & Indian pond heron & Ardeidae & Least concern & Residential & Aquatic & $2^{\text {nd }}$ most dominant \\
\hline Saxicoloidesfulicatus & Indian Robin & Muscicapidae & Least concern & Residential & Terrestrial & least abundant \\
\hline Coraciasbenghalensis & Indian Roller & Coraciidae & Least concern & Residential & Terrestrial & least abundant \\
\hline Ardeaintermedia & Intermediate egret & Ardeidae & Least concern & Residential & Aquatic & $2^{\text {nd }}$ most dominant \\
\hline Microcarboniger & Little cormorant & Podicipedidae & Least concern & Residential & & most dominant \\
\hline Egrettagarzetta & Little egret & Ardeidae & Least concern & Residential & Aquatic & sub-dominant \\
\hline Tachybaptusruficollis & Little grebe & Podicipedidae & Least concern & Residential & Aquatic & $2^{\text {nd }}$ most dominant \\
\hline Charadriusdubius & Little ringed plover & Charadriidae & Least concern & Residential & Terrestrial & sub-dominant \\
\hline Calidrisminuta & Little stint & Scolopacidae & Least concern & Residential & Aquatic & Sub-dominant \\
\hline Anasplatyrhynchos & Mallard & Anatidae & Least concern & Migratory & Aquatic & $2^{\text {nd }}$ most dominant \\
\hline Anasacuta & Northern pintail & Anatidae & Least concern & Migratory & Aquatic & most dominant \\
\hline Sylvia hortensis & Orphean Warbler & Sylviidae & Least concern & Residential & Terrestrial & least abundant \\
\hline Hydrophasianuschirurgus & Pheasant tail Jacana & Jacanidae & Least concern & Residential & Aquatic & least abundant \\
\hline Ardeapurpurea & Purple heron & Ardeidae & Least concern & Residential & Aquatic & sub-dominant \\
\hline Cinnyrisasiaticus & Purple Sunbird & Nectariniidae & Least concern & Residential & Terrestrial & least abundant \\
\hline Laniuscollurio & Red Backed Shrike & Laniidae & Least concern & Residential & Terrestrial & least abundant \\
\hline Streptopeliatranquebarica & Red Collared Dove & Columbidae & Least concern & Residential & Terrestrial & least abundant \\
\hline Tringatotanus & Red shank & Scolopacidae & Least concern & Residential & Aquatic & sub-dominant \\
\hline Vanellusindicus & Red wattled lapwing & Charadriidae & Least concern & Residential & Terrestrial & $2^{\text {nd }}$ most dominant \\
\hline Pycnonotuscafer & Red-Vented bulbul & Pycnonotidae & Least concern & Residential & Terrestrial & least abundant \\
\hline Vanellusindicus & Red-Wattled Lapwing & Charadriidae & Least concern & Residential & Terrestrial & least abundant \\
\hline Cercotrichasgalactotes & Rufous-Tailed Scrub Robin & Muscicapidae & Least concern & Residential & Terrestrial & least abundant \\
\hline Accipiter badius & Shikra & Accipitridae & Least concern & Residential & Terrestrial & least abundant \\
\hline Passer hispaniolensis & Spanish Sparrow & Passeridae & Least concern & Residential & Terrestrial & least abundant \\
\hline Spilopeliachinensis & Spotted Dove & Columbidae & Least concern & Residential & Terrestrial & least abundant \\
\hline Vanellusleucurus & White-tailed plover & Charadriidae & Least concern & Residential & Terrestrial & sub-dominant \\
\hline
\end{tabular}

Journal of Innovative Sciences

December 2021 | Volume 7 | Issue 2 | Page 240 
2008-2014, and they recorded 171 species of birds (Bibi et al., 2016).

At Taunsa barrage wildlife sanctuary, 58,598 birds recorded, which belonging to 53 families and 171 species (Bibi and Ali, 2013) and Akhtar reported 126 bird species from chashma, 115 from mammal lake, 110 from rangpur while 103 were reported from uchali lake (Ali et al., 2007). In another study, 110 species of bird population were recorded at Taunsa Barrage wildlife sanctuary (Ali et al., 2011). At Trimmubarrage, a total of 9,699 birds, 89 species belonging to 39 families, and 15 orders were recorded during 2004-2005 (Shahid et al., 2009). At Uchalilake, 11 species were visited in 2010, with their total number up to 1,139. In 2011 total, 34 species with a population of 18,606 birds were recorded there (Arshad et al., 2014).

Birds occurrence at Jiwani coastal wetlands in 2008 the total number of species were 109,77 recorded as migratory birds and 32 as residents Studies have been conducted on same parameters in different parts of the world by other researchers as the birds of Pakistan studied by Robert (1991-92). One hundred twenty species were recorded; 46 as winter visitor;40 species were recorded as residents; 15 as summer visitor; summer breeders as five species; irregular year-round visitors were recorded as 11 species; 2 was recorded as vagrant and spring visitor was only one species recorded, Grimmett et al. (1998). A total of 101 birds species; 35 winter visitors; residents 39 species; 15 were summer visitors; 2 were recorded as summer breeders; 5 species were recorded as irregular year-round visitors and six species as a vagrant, Mirza (2007) were recorded 84 species at Jiwanicoastak wetland, residential birds were 33 species, 25 species of them were winter visitors, summer visitors were 11 species, 4 were summer breeder, 9 were an irregular year-round visitor and 2 of them were vagrant. During the year 2008, 110 bird species were recorded at Taunsa barrage. The avifauna observed during this study belonged to 45 families. Among 110 species, 66 were residents, eight were breeding residents, winter visitors were 34 , and summer visitors were only 2.

According to the field survey from March 2009 to December 2009 (Tables 2), a total of $(\mathrm{N}=176579)$ individuals were recorded from Karachi University and Safari Park. Out of which (n1=119986) (Table
2) individuals were recorded from Karachi University. Of which 10063 individuals were recorded during March, 10469 during April, 10562 during May, 10997 during June, 11552 during July, 12376 during August, 13768 during September, 13593 during October, 13738 during November, and 12868 during December.

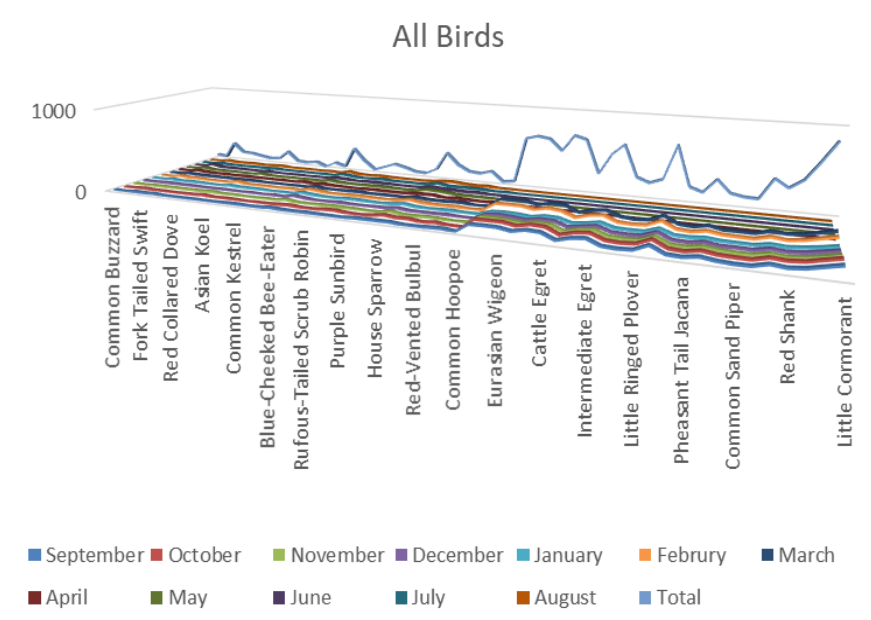

Figure 1: Great variety of birds fauna at dhapchapak wetland and forest.

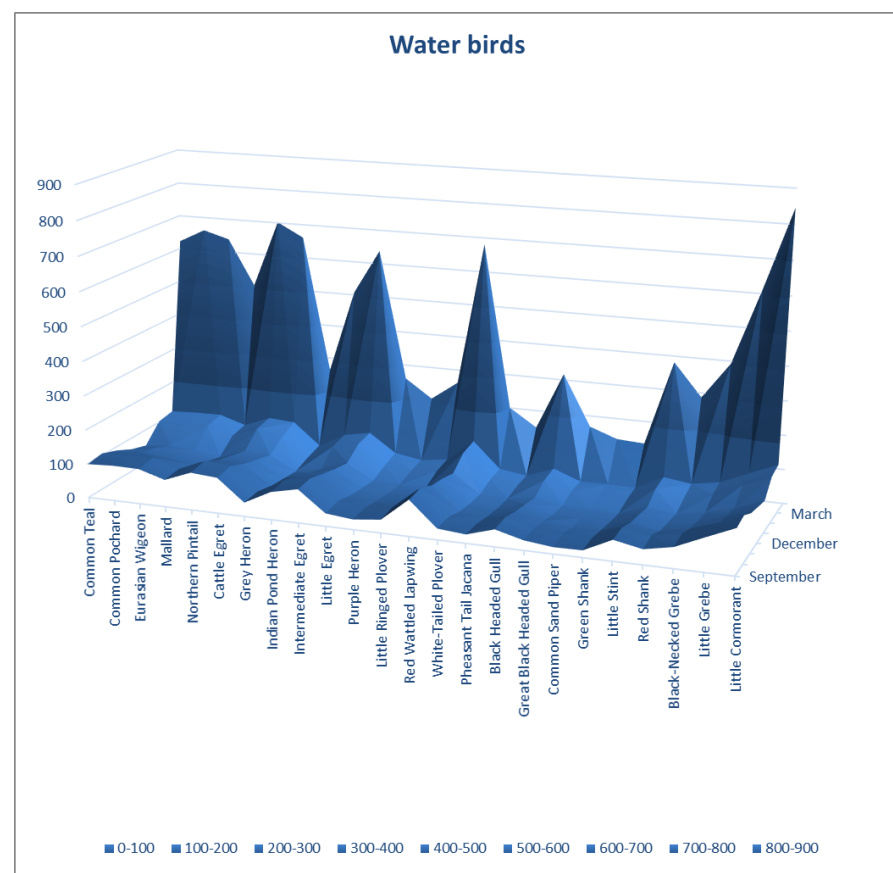

Figure 2: The total number of water birds recorded monthly wise during 2017 to 2019.

While from the Wild habitat of Safari Park area, a total of $(\mathrm{n} 2=56593)$ individuals of birds were recorded. Out of these 4838 individuals were recorded during March, 4500 during April, 4675 during May, 4910 during June, 6150 during July, 6532 during August, 6525 during September, 7069 during October, 5842 November, and 5552 during December. 


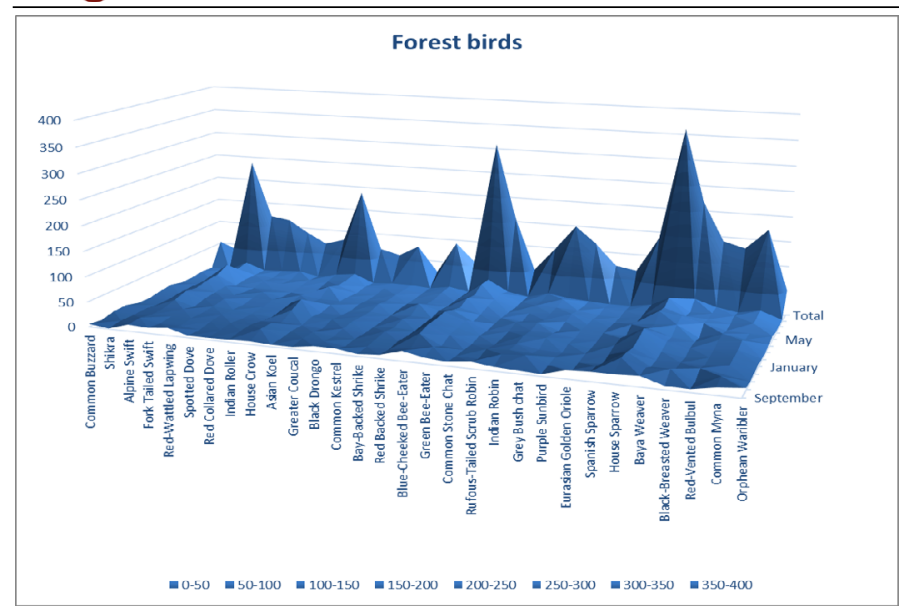

Figure 3: The total number of forest birds recorded monthly wise during 2017 to 2019 .

\section{Acknowledgments}

Throughout the writing of this thesis I have received a great deal of support and assistance.

First of all would like to express our gratitude to Almighty ALLAH to enabling us to complete this research.

We would like to thank our supervisor Inam Ullah, whose expertise was invaluable in formulating the research questions and methodology. Your insightful feedback pushed me to sharpen my thinking and brought my work to a higher level.

Would particularly like to single out supervisor Ruqia Bibi and Najam-un-Nisa. We want to thank you for your patient support and for all of the opportunities was given to further our research, for their valuable guidance throughout studies. You provided us with the tools that we needed to choose the right direction and successfully complete our dissertation.

In addition, would like to thank our parents for their wise counsel and sympathetic ear. You are always there for us. Finally, this dissertation without saima,balqees riaz, uzma islma khan, iqra maheen, bushra khalil couldn't complete who stimulating discussions as well as happy distractions to rest mind outside of our research and helped a lot in writing the research thesis and provide guidance throughout studies.

\section{Novelty Statement}

The work was conducted first time at this riverine forest area, in which the number of birds species were

explained very clearly and the reasons of declining of birds also discussed.

\section{Author's Contribution}

Inam Ullah designed the data. Ruqia Bibi and Najam Un Nisa reviewed the manuscript. Balqees Riaz wrote the introduction, Uzma Islam Khan and Iqra Maheen wrote the discussion, while Bushra Khalil and Saima wrote the results of the manuscript. Methodology designed by all the authors.

\section{Conflict of interest}

The authors have declared no conflict of interest.

\section{References}

Ali, A., Altaf, M., Khan, M.S.H., Khan, A.M., Ashraf, S., and Chattha, S.A., 2017. Avifauna diversity along the coastline of Banbhore (Gharo creek), district Thatta, Sindh, Pakistan. Journal of Wildlife and Ecology, 1(1): 8-16.

Ali, Z., 2005. Ecology, distribution and conservation of migratory birds at Uchalli Wetlands Complex, Punjab, Pakistan. Diss. University of Punjab.

Ali, Z., 2011. Comparative avian faunal diversity of Jiwani coastal wetlands and taunsa barrage wildlife sanctuary, Pakistan. Journal of Animal and Plant Sciences, 21(2): 381-387.

Arshad, M., 2014. Avifauna studies in co-relation with alteration in climatic patterns and hydrology of Uchalli Lake, Punjab, Pakistan. Pakistan Journal of Zoology, 46(2): 503-515.

Bibi, F., Qaisrani, S.N., and Akhtar, M., 2016. Assessment of population trends of birds at Taunsa Barrage Wildlife Sanctuary, Pakistan. Biologia, 62: 201-210.

Bibi, F., and Ali, Z., 2013. Measurement of diversity indices of avian communities at Taunsa Barrage Wildlife Sanctuary, Pakistan. The Journal of Animal and Plant Sciences, 23(2): 469-474.

Brenner, J., 2010. An assessment of the non-market value of the ecosystem services provided by the Catalan coastal zone, Spain. Ocean and Coastal Management, 53(1): 27-38.

Buckton, S., 2007. Managing wetlands for sustainable livelihoods at Koshi Tappu. Danphe 16 (1): 12-13.

Daniel, K.W., Chu, I., Connie, Y.H., Leung, M.G., Priscilla, H.J., Erica M.N., Tsemay, M.T., Yi, 
G., Joseph, S.M., Peiris, L., and Poon, L.M. 2011. Avian coronavirus in wild aquatic birds. 2011 Sep 28

Gamfeldt, L., Snäll, T., Bagchi, R., Jonsson, M., Gustafsson, L., Kjellander, P., Ruiz-Jaen, M.C., Fröberg, M., Stendahl, J., Philipson, C.D., Mikusiński, G., Andersson, E., Westerlund, B., Andrén, H., Moberg, F., Moen, and J., Bengtsson J. 2013. Higher levels of multiple ecosystem services are found in forests with more tree species. Nature Communications, 4: 1340. doi: http://dx.doi.org/10.1038/ncomms2328.

Girmmett, R., G., Inskipp, C., and Inskipp T., 1998. Birds of the Indian Subcontinent.

Kazmierczak, K., 2000. A field guide to the birds of India, Sri Lanka, Pakistan, Nepal, Bhutan, Bangladesh, and the Maldives. Om Book Service, 2000.

Mahmood, T., Luqman U.K., and Muhammad N., 2021. Diversity and abundance of Avifauna of Manglot Wildlife Park, Nowshera District, Khyber Pakhtunkhwa, Pakistan.

Mirza, Z.B., and Wasiq, H., 2007. A field guide to birds of Pakistan.

Pei, N., Wang, C., Jin, J., Jia, B., Chen, B., Qie, G., and Zhang, Z., 2018. Long-term affores- tation efforts increase bird species diversity in Beijing, China. Urban Forestry and Urban Greening, 29: 88-95. https://doi.org/10.1016/j. ufug.2017.11.007

Rais, M., 2011. Bird diversity and conservation at Kallar Kahar Lake with special reference to water birds." Pakistan J. Zool. 43(4): 673-681.

Roberts, Tom, J., 1991. The Birds of Pakistan: Passeriformes: Pittas to Buntings. Vol. 2. Oxford University Press, 1991.

Shahid, M., 2009. Diversity of avifauna of Trimmu Barrage, District Jhang, Punjab, Pakistan. Pakistan Journal of Zoology, 41.1.

Whelan, Christopher, J., Daniel, G., Wenny., and Robert, J.M.2008. Ecosystem services provided by birds. Annals of the New York academy of sciences, 1134 (1): 25-60.

Woldemariam, W., Mekonnen, T., Morrison, K., and Aticho, A., 2018. Assessment of wetland flora and avifauna species diversity in Kafa Zone, Southwestern Ethiopia. Journal of $\mathrm{Asia}_{\mathrm{Pa}} \mathrm{P}$ cific Biodiversity, 11(4): 494-502. https://doi. org/10.1016/j.japb.2018.08.003

Woodcock, M., 1980. Collins Hand Guide to the Birds of Indian Subcontinent. London E. H. SIMPSON, NATURE, April 30, 1949 Vol. 163 